\title{
Winstats en el aprendizaje de estadística en los estudiantes de informática de la Universidad Enrique Guzmán y Valle -Perú
}

\section{Winstats in the learning of statistics in computer science students at the Enrique Guzmán y Valle University- Peru}

Lolo José Caballero-Cifuentes

Universidad Nacional de Educación 'Enrique Guzmán y Valle', Lima, Perú lcaballero@une.edu.pe

(D) https://orcid.org/0000-0002-9764-1327

Guillermo Pastor Morales-Romero

Universidad Nacional de Educación 'Enrique Guzmán y Valle', Lima, Perú gmorales@une.edu.pe

(i) https://orcid.org/0000-0002-5686-7661

Nicéforo Ladislao Trinidad-Loli

Universidad Nacional de Educación 'Enrique Guzmán y Valle', Lima, Perú ntrinidad@une.edu.pe

https://orcid.org/0000-0002-0630-6348

Adrián Quispe-Andía

Universidad Nacional de Educación 'Enrique Guzmán y Valle', Lima, Perú aquispe@une.edu.pe

https://orcid.org/0000-0001-6894-2799

Recepción: 01/08/2021 | Aceptación: 09/11/2021 | Publicación: 25/11/2021

Cómo citar (APA, séptima edición):

Caballero-Cifuentes, L. J., Morales-Romero, G. P., Trinidad-Loli, N.L., y Quispe-Andía, A. (2021). Winstats en el aprendizaje de estadística en los estudiantes de informática de la Universidad Enrique Guzmán y Valle-Perú. INNOVA Research Journal, 6(3.2), 32-46. https://doi.org/10.33890/innova.v6.n3.2.2021.1867

\section{Resumen}

En el presente artículo da a conocer el efecto del software Winstats en el aprendizaje de estadística y determinar el nivel de aprendizaje en la Facultad de Ciencias, mediante un análisis teórico y práctico. La metodología de la investigación es de tipo aplicada, enfoque cuantitativo, diseño cuasi 
Winstats en el aprendizaje de estadística en los estudiantes de informática de la Universidad Enrique Guzmán y Valle-Perú

experimental y método empírico teórico. Para determinar la muestra se utilizó el método estadístico no probabilístico, siendo la muestra de 25 estudiantes del grupo experimental y 25 de control; a quienes se les aplicó una prueba de conocimientos validada por juicio de expertos y de confiabilidad con la técnica estadística de dos mitades, sub técnica Spearman Brown, obteniendo los coeficientes de 0,85 y 0,86 respectivamente, para cada uno de los cuestionarios aplicados. Para la prueba de hipótesis se utilizó el t Student para muestras independientes, asumiéndose un nivel de confianza $95 \%$ y nivel de significancia 5\%. Los resultados después del experimento fueron favorables para el grupo experimental en sus dimensiones conceptual y procedimental, obteniéndose promedios con mayor puntaje de 15,60 en comparación al grupo control 12,16. En conclusión, se logró demostrar que si hubo efecto significativo del Software Winstats en el aprendizaje de estadística.

Palabras claves: software; Winstats; aprendizaje; estadística; educación.

\begin{abstract}
In this article we present the effect of the Winstats software on the learning of statistics and determine the level of learning in the Faculty of Sciences, through a theoretical and practical analysis. The research methodology is applied, quantitative approach, quasi-experimental design and theoretical empirical method. To determine the sample, the non-probabilistic statistical method was used, the sample being 25 students from the experimental group and 25 from the control group; To whom a knowledge test validated by expert judgment and reliability was applied with the statistical technique of two halves, the Spearman Brown sub technique, obtaining the coefficients of 0.85 and 0.86 respectively, for each of the applied questionnaires. For the hypothesis test, the Student's t test was used for independent samples, assuming a 95\% confidence level and a 5\% level of significance. The results after the experiment were favorable for the experimental group in its conceptual and procedural dimensions, obtaining averages with a higher score of 15.60 compared to the control group 12,16. In conclusion, it was possible to demonstrate that there was a significant effect of the Winstats Software on the learning of statistics.
\end{abstract}

Keywords: software; Winstats; learning; statistics; education.

\title{
Introducción
}

La estadística juega un papel importante en la investigación educativa, frente a los tópicos, métodos, técnicas y análisis de datos útiles para la investigación científica; por consiguiente, es ineludible que los docentes universitarios conozcan y apliquen diversos softwares estadísticos en el proceso de enseñanza aprendizaje. En el contexto actual, mejorar los niveles de aprendizaje implica para el educador un cambio paradigmático, al aplicar las nuevas tecnologías de la información y comunicación, encontrándonos constantemente en la búsqueda de nuevas estrategias de aprendizaje con el propósito de superar los métodos tradicionales.

Enseñar estadística no solo es resolver problemas en forma matemática, sino también es necesaria la ayuda de softwares especializados para procesar datos, analizar, presentar la información, interpretar y tomar decisiones pertinentes. Las tecnologías de información y comunicación son aplicadas en distintas disciplinas y en diferentes niveles educativos, 
respondiendo a la demanda que la era digital plantea la sociedad en general y a la educación en particular.

En este escenario se plantea la necesidad pertinente para que los estudiantes de la Facultad de Ciencias de la Universidad Enrique Guzmán y Valle, Perú, estén en condiciones de aprender y aprovechar los diferentes recursos tecnológicos y herramientas estadísticas necesarias para resolver trabajos relacionados a la investigación y al campo educativo.

En atención a esta problemática se considera conveniente realizar el estudio con las variables software estadístico y Winstats en el aprendizaje de estadística en los aspectos análisis descriptivo de datos, análisis inferencial, validez y confiablidad, desarrollándose en base a las dimensiones conceptual y procedimental aplicada a un grupo experimental en comparación con el aprendizaje tradicional grupo control, el procedimiento se desarrolló durante 12 semanas, 2 horas estudio.

El objetivo general fue determinar el efecto del software Winstats en el aprendizaje de estadística, en los estudiantes de la especialidad de informática de la Facultad de Ciencias de la Universidad Enrique Guzmán y Valle, Perú, 2019. Los objetivos específicos fueron: Conocer el efecto del Software Winstats en el aprendizaje de estadística en su dimensión conceptual. Determinar el efecto del Software Winstats en el aprendizaje de estadística en su dimensión procedimental. Establecer diferencia entre el nivel de aprendizaje de estadística, en los grupos control y experimental después de haber aplicado el Software Winstats. Así mismo, la hipótesis general planteada es: Existe efecto significativo del Software Winstats en el aprendizaje de estadística, en los estudiantes de la especialidad de informática.

\section{Marco Teórico}

El presente estudio se basó en los siguientes antecedentes:

Álvarez y Solís (2019) investigaron sobre uso de GeoGebra y el aprendizaje de la estadística descriptiva. Tipo de investigación básica aplicada con diseño cuasi experimental. Desarrolló en 91 estudiantes, 46 para el grupo experimental, 45 en el control, a quienes aplicó una prueba de conocimientos validada por la técnica de juicio de expertos y la confiabilidad con el coeficiente de alfa de Cronbach. Los resultados le indicaron una media aritmética para el postest grupo experimental de 14,00 frente a la media aritmética del postest grupo de control de 10,00. El contraste de la prueba lo realizó con el estadístico Z para dos muestras, con ayuda del Excel los datos fueron de 6,00 para z-calculado en comparación con el z-critico 1,64. Esta información le dio la certeza para concluir que hubo influencia del uso del software GeoGebra en el aprendizaje de la estadística descriptiva para estudiantes del cuarto grado de educación secundaria de la Institución Educativa Daniel Alcides Carrión de Cerro de Pasco, Perú, 2018. 
Winstats en el aprendizaje de estadística en los estudiantes de informática de la Universidad Enrique Guzmán y Valle-Perú

Medina (2019) investigó sobre la aplicación del software SPSS en el proceso de enseñanza de estadística. tipo de investigación aplicada, enfoque cuantitativo, el diseño cuasi experimental conformado por dos grupos uno control y el otro experimental. La muestra 45 estudiantes para ambos grupos. Los instrumentos fueron cuestionarios tipo Likert validados con la prueba de Alpha de Cronbach, los coeficientes obtenidos fueron muy buenos y altos, 0.914. Los resultados han mostrado porcentajes favorables $46.57 \%$ al presentar tablas, gráficos, análisis descriptivo e inferencial de datos. La prueba de hipótesis contrastó con el estadístico no paramétrico de wilcoxon, La evidencia precisa un cambio pertinente de las dimensiones y las competencias del proceso de enseñanza aprendizaje de Estadística en los estudiantes del segundo ciclo de la Escuela Profesional de Turismo y Hotelería de la Facultad de Ciencias de la Comunicación, Turismo y Psicología de la Universidad de San Martín de Porres, Lima, Perú.

Estrada (2002) investigó sobre el Análisis de las Actitudes y Conocimientos Estadísticos en la formación del profesorado, desarrollado por la universidad de Barcelona. España. El estudio exploratorio, método empírico teórico con diseño descriptivo correlacional. ha tenido dos fases y enfoques diferentes actitudes hacia la Estadística, comparando los profesores en formación y en ejercicio, el nivel de la enseñanza primaria con una muestra reducida. Sus resultados indicaron la estrecha relación que existente entre las actitudes y los conocimientos hacia la Estadística. Los resultados también indicaron que sólo el número de años de estudio tiene un efecto estadísticamente significativo sobre la puntuación media en la escala de actitudes.

Torres (2019) investigó el SIMSTAT en el aprendizaje de estadística en los estudiantes de la Universidad Nacional de Educación en Lima Perú. tipo de investigación aplicada con enfoque cuantitativo, diseño fue cuasi experimental y el método es empírico teórico. La muestra lo determinó mediante el muestreo no probabilístico, 28 estudiantes para el grupo experimental y 28 para el control. los instrumentos fueron dos pruebas de conocimientos previamente validados por la técnica de juicio de expertos con calificaciones porcentuales de 85 y $82.25 \%$ y la confiabilidad del instrumento mediante la técnica estadística de dos mitades sub técnica spearman Brown obteniéndose coeficientes de confiabilidad muy alta de 0,82 y 0.86 respectivamente. Los resultados le indicaron promedios altos para el grupo experimental después de aplicar el SIMSTAT a nivel descriptivo en sus dimensione: Análisis descriptivos de datos, Confiabilidad-validez y Análisis inferencia. La prueba de hipótesis lo realizó con el estadístico T -Student para muestras independientes; obteniéndose valores inferiores con ayuda del SPSS del p-valor 0.01 en comparación del nivel de significancia 0,05 . En conclusión, logró determinar y conocer el efecto de software SIMSTAT en el aprendizaje de la estadística, cuyos niveles de aprendizaje observados después del experimento fueron altos, con tendencia a subir en comparación del grupo control que obtuvieron niveles regulares con tendencia a bajar.

Referido a las bases teóricas, se explica el desarrollo de las variables: Winstats y aprendizaje de estadística.

Winstats, es sencilla, dinámica, interactiva y completa herramienta para la realización de cálculos y representaciones estadísticas, con una o dos variables, para una variable permite 
introducir o importar los datos de un texto u hoja de cálculo, nos da de forma automática todos los parámetros estadísticos: número de datos, valor mínimo, cuartiles, mediana, máximo valor, deciles, percentiles, media aritmética, rango, rango intercuartílico, desviación media, desviación típica, desviación estándar, también nos permite representar gráficamente los datos mediante diagramas de cajas, histogramas, ajuste a la normal. Para dos variables: permite dibuja la nube de puntos representándolas, realiza cálculos directamente de la ecuación de la recta de regresión de Y sobre X. Admite regresiones polinómicas, exponenciales, logarítmicas. Además, tiene la ventaja de interpolar, extrapolar, estimar valores en función de una de las variables, calcula distancias de un punto a la recta de regresión, representa mínimos cuadrados, cálculos con probabilidades y distintas distribuciones. En su interfaz incluye hasta 12 experimentos distintos: urnas con bolas de colores, extracción de cartas, lanzamientos de agujas, de monedas, dianas, ruletas numéricas, generación de números aleatorios; realizando el estudio estadístico y la presentación de los resultados obtenidos. (Paris, 1996)

Los nuevos profesionales deben dominar técnicas, procedimientos o herramientas, más que conocimientos teóricos o premisas fundamentales de una disciplina. Esto significa poner en práctica el saber hacer. (Bauman, 2013). Las computadoras proveen el aprendizaje dinámico e interactivo que permiten la rápida visualización de situaciones problemáticas, la posibilidad de visualizar gráficamente conceptos teóricos como también las diferentes variables que intervienen en la resolución de problemas, favorece al aprendizaje de los alumnos (Sanchez,1998).

Para llegar a comprender que es la estadística, es necesario definir previamente qué se entiende en la actualidad por ciencia estadística. La estadística es el estudio de los datos cuantitativos de la población, de los recursos naturales e industriales, del tráfico o de cualquier otra manifestación de las sociedades humanas. Bien, ésta es lo que algunos autores llaman "concepción profana" de la estadística o, dicho de otro modo, lo que consideran la mayoría de las personas que no se han especializado en esta ciencia. En realidad, se debe reconocer que operaciones tales como la recopilación de grandes masas de datos, su presentación en forma de cuadros, tablas, gráficos, el cálculo de promedios, porcentajes, variaciones, etc., forman parte de nuestra ciencia, y de hecho constituyen la denominada "estadística descriptiva". Pero todo aquello no queda ahí, sino que hay en realidad otras cuestiones generales más complejas de mayor alcance y profundidad (García, 2013).

Para Caballero, Quivio, Morales, Cajavilca, Gutiérrez y Vargas (2016), manifiestan que la estadística, una ciencia aplicada, nos proporciona un conjunto de métodos, técnicas y modelos para recopilar, organizar, resumir, presentar, analizar, hacer predicciones e interpretar datos para tomar decisiones sobre determinados hechos o fenómenos de estudio. Para Salazar, Del Castillo (2018), manifiestan que la estadística es la ciencia que se encarga de la recolección, ordenamiento, representación, análisis e interpretación de datos generados en una investigación, hechos, individuos o grupos, para poder deducir las conclusiones y estimaciones futuras. 


\section{Metodología}

El Enfoque de investigación fue de corte cuantitativo, dado que se contrasta cuantitativamente la aplicación del Software Winstats en el campo del aprendizaje de la estadística en los estudiantes de nivel universitario. El método empleado en la investigación es empírico y teórico con diseño cuasi experimental, debido a que se aplica una propuesta educativa basada en el uso del Software Winstats de aplicación informática como estrategia metodológica a un grupo experimental. Para la elección del diseño se tomó en cuenta que los estudiantes no son asignados al azar a los grupos, sino que dichos grupos ya están formados antes del experimento, son grupos intactos (Hernández, Fernández y Baptista, 2014).

El tipo de investigación es experimental en función a la aplicación del Software Winstats en el campo del aprendizaje de la estadística en los estudiantes de nivel universitario. El diseño se esquematizó de la forma:

\begin{tabular}{cccc}
\multicolumn{3}{c}{ Pre prueba } & Pos prueba \\
GC: & $\mathrm{O}_{1}$ & - & $\mathrm{O}_{3}$ \\
$\mathrm{GE}:$ & $\mathrm{O}_{2}$ & $\mathrm{X}$ & $\mathrm{O}_{4}$ \\
$\mathrm{GC}:$ & Grupo de control, enseñanza sin Software Winstats \\
$\mathrm{GE}:$ & Grupo experimental, enseñanza con el Software Winstats \\
- - & Tratamiento sin el Software Winstats \\
$\mathrm{X}:$ & Tratamiento con el Software Winstats \\
$\mathrm{O}_{1}:$ & Observación inicial del Grupo Control \\
$\mathrm{O}_{2:}$ & Observación inicial del Grupo Experimental \\
$\mathrm{O}_{3}:$ & Observación final del Grupo Control \\
$\mathrm{O}_{4}:$ & Observación final del Grupo Experimental
\end{tabular}

En cuanto al procedimiento del experimento para el estudio se seleccionó dos secciones, uno de control y el otro experimental. Se aplicó en un primer momento una prueba inicial a ambos grupos con la finalidad de conocer el nivel de aprendizaje de estadística. Luego en ambos grupos se desarrollaron contenidos teóricos y prácticos sobre cálculos, representaciones estadísticas, análisis de datos a nivel descriptivo e inferencial y probabilidades. Al grupo experimental a diferencia del grupo control se le aplicó en el desarrollo de las clases el Software Winstats durante el ciclo de estudios. Finalizado el periodo académico se aplicó a ambos grupos una segunda prueba final con el fin de obtener resultados que nos indique el nivel de aprendizaje de los estudiantes en la asignatura de estadística.

La población de estudio lo constituyeron 116 estudiantes del cuarto ciclo de la Facultad de Ciencias de la Universidad Enrique Guzmán y Valle La Cantuta. Ver tabla 1 


\section{Tabla 1}

Distribución de la Población

\begin{tabular}{lc}
\hline Universidad Enrique Guzmán y Valle la Cantuta & $\mathbf{N}^{\circ}$ Estudiantes \\
\hline Sección c6 & 25 \\
Sección c5 & 34 \\
Sección c1 & 32 \\
Sección c 9 & 25 \\
Total & $\mathbf{1 1 6}$ \\
\hline
\end{tabular}

Fuente: Elaboración propia

Para muestra de la investigación se consideró el muestreo del método no probabilístico. Para ello, se necesitó dos grupos de trabajo que se tomara, en forma arbitraria o intencional, puesto que, los grupos previamente están establecidos. Se tomó en cuenta los criterios como: el nivel educativo para no alterar la investigación, el nivel socioeconómico, la misma asignatura y el mismo ciclo académico. Ver tabla 2

\section{Tabla 2}

Distribución de la muestra

\begin{tabular}{lccc}
\hline & Universidad Enrique Guzmán y Valle. Facultad de ciencias \\
Grupos & Secciones o bloques & Estudiantes \\
\hline Control & Sección c6 & 25 \\
Experimental & Sección cl & 25 \\
& \multirow{2}{*}{ Total } & & 50 \\
\hline
\end{tabular}

Fuente: Elaboración propia

\section{Tabla 3}

Baremo para niveles de aprendizaje de acuerdo con los promedios

\begin{tabular}{ll}
\hline Promedios & Niveles de aprendizaje \\
\hline 18 a 20 & Excelente \\
15 a 17 & Bueno \\
11 a 14 & Regular \\
6 a 10 & Malo \\
0 a 5 & Pésimo \\
\hline
\end{tabular}

Fuente: Elaboración propia 
La tabla 3 fue diseñada en base a la obtención de resultados de prueba vigesimal administrada, con el objetivo de interpretar los niveles de aprendizaje de estadística en los estudiantes para los grupos control y experimental.

\section{Tabla 4}

Comparación de promedios de ambos momentos grupo experimental y control

\begin{tabular}{lllll}
\hline \multicolumn{1}{c}{ Grupos } & & Pre prueba & & Pos prueba \\
\cline { 1 - 1 } Grupo Control & & 12,16 & 13,24 \\
Grupo experimental & 13,28 & 15,6 \\
\hline
\end{tabular}

Fuente: Elaboración propia

En la tabla 4 el resultado de la aplicación de las pruebas revela que, en la pre prueba, es decir, antes del experimento, el promedio para el grupo control fue de 12,06, mientras para el grupo experimental 13, 28, aparentemente no muy significativo. Luego de aplicar el software estadístico Winstats al grupo experimental en el pos prueba se observa un promedio para el grupo control de 13, 24, grupo experimental 15,60. Predominando el promedio en el pos prueba para el grupo experimental en comparación al promedio del grupo control. En base a estos resultados se afirma con certeza que la aplicación del software Winstats tiene un efecto muy significativo en el aprendizaje de estadística en sus dimensiones conceptual y procedimental. El nivel que se logró es bueno con tendencia a subir después del experimento.

\section{Figura 1}

Análisis de comparación de promedios de ambos momentos: grupo experimental y control

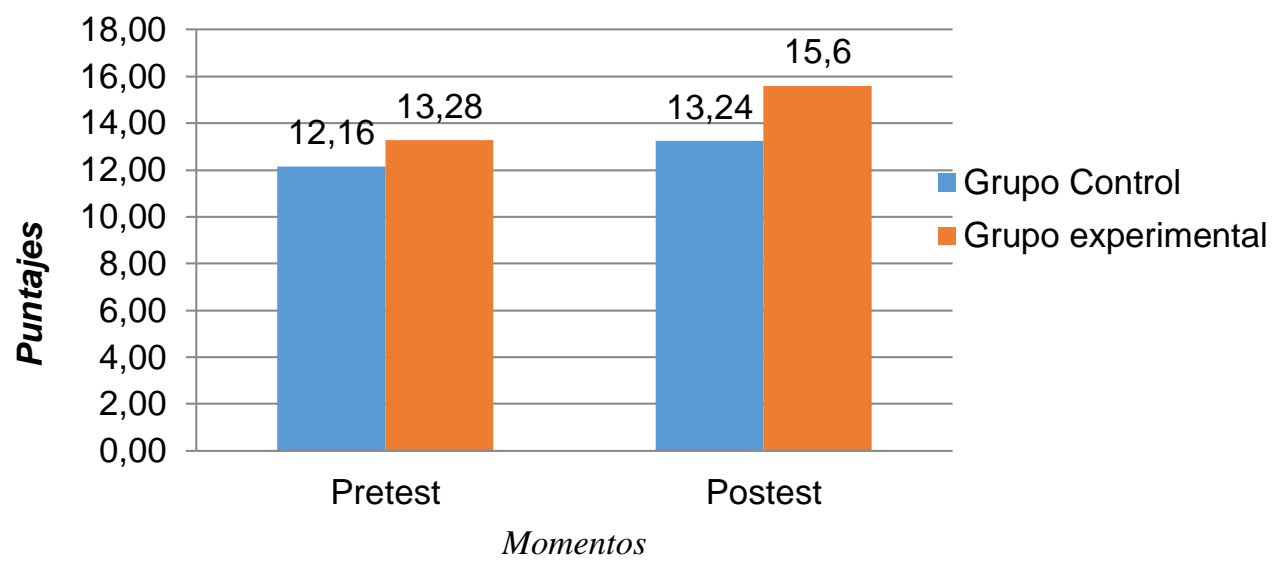




\section{Tabla 5}

Estadísticos de la variable dependiente: Aprendizaje de estadística

\begin{tabular}{|c|c|c|c|c|c|}
\hline & & $\begin{array}{l}\text { Pre prueba } \\
\text { grupo Control }\end{array}$ & $\begin{array}{l}\text { Pos prueba } \\
\text { grupo } \\
\text { Control }\end{array}$ & $\begin{array}{c}\text { Pre prueba } \\
\text { grupo } \\
\text { Experimental }\end{array}$ & $\begin{array}{c}\text { Pos prueba grupo } \\
\text { Experimental }\end{array}$ \\
\hline \multirow[t]{2}{*}{$N$} & Válido & 25 & 25 & 25 & 25 \\
\hline & Perdidos & 0 & 0 & 0 & 0 \\
\hline \multicolumn{2}{|c|}{ Media } & 12.16 & 13.24 & 13.28 & 15.60 \\
\hline \multicolumn{2}{|c|}{ Mediana } & 12.00 & 13.00 & 13.00 & 15.00 \\
\hline \multicolumn{2}{|c|}{ Desviación estándar } & 1.344 & 1.615 & 2.151 & 1.732 \\
\hline \multicolumn{2}{|c|}{ Varianza } & 1.807 & 2.607 & 4.627 & 3.000 \\
\hline \multicolumn{2}{|c|}{ Mínimo } & 9 & 11 & 10 & 12 \\
\hline \multicolumn{2}{|c|}{ Máximo } & 15 & 18 & 17 & 18 \\
\hline
\end{tabular}

Fuente: Elaboración propia

En la tabla 5 se observó los estadísticos para la variable dependiente aprendizaje de estadística, con una muestra de 25 estudiantes por grupo, el promedio para el pre prueba grupo control 12,06, grupo experimental 13, 28; para el pos prueba grupo control 13,24 y experimental 15, 60. Siendo favorable para grupo experimental. Con respecto a la mediana se observa en el pre prueba grupo experimental el 50\% de estudiantes lograron obtener un nivel de aprendizaje inferior igual a $13.00 \mathrm{y}$ el resto superior, a diferencia en la pos prueba un nivel de aprendizaje inferior igual a 15,00 y el resto de las estudiantes superior a 15.00, con una desviación típica de 1,7 respecto de la media. Estos resultados nos indican que hubo un nivel bueno en el aprendizaje de estadística después de aplicar el Winstats.

\section{Contraste de prueba de hipótesis}

Las hipótesis de investigación planteadas para determinar el efecto de software Winstats en el aprendizaje de estadística, fue determinada con el método estadístico, paramétrico t Student para muestras independientes por las razones siguientes: distribución de datos normal, muestra menor que 30 y diseño cuasi experimental para comparar promedios por grupo. Hipótesis General. Planteamiento de la hipótesis. Hipótesis nula: No existe efecto significativo del software Winstats en el aprendizaje de estadística, en los estudiantes de la especialidad de informática de la Facultad de Ciencias de la Universidad Enrique Guzmán y Valle. Hipótesis general: Existe efecto significativo del software Winstats en el aprendizaje de estadística. Nivel de confianza al 95\% nivel de significancia de 5\%, con ayuda del software estadístico SPSS se obtuvo los resultados del contraste: 
Winstats en el aprendizaje de estadística en los estudiantes de informática de la Universidad Enrique Guzmán y Valle-Perú

Tabla 6

Prueba de hipótesis para determinar el efecto del software Winstats en el aprendizaje de estadística

\begin{tabular}{|c|c|c|c|c|c|c|c|c|c|c|}
\hline & & \multicolumn{9}{|c|}{ Prueba t para la igualdad de medias } \\
\hline & & \multirow[b]{2}{*}{$\mathbf{F}$} & \multirow[b]{2}{*}{$\begin{array}{l}\mathrm{Si} \\
\text { g. }\end{array}$} & \multirow[b]{2}{*}{$\mathbf{t}$} & \multirow[b]{2}{*}{ gl } & \multirow{2}{*}{$\begin{array}{l}\text { Sig. } \\
\text { (bilater } \\
\text { al) }\end{array}$} & \multirow{2}{*}{$\begin{array}{l}\text { Diferen- } \\
\text { cia de } \\
\text { medias }\end{array}$} & \multirow{2}{*}{$\begin{array}{l}\text { Diferen- } \\
\text { cia de } \\
\text { error } \\
\text { estándar }\end{array}$} & \multicolumn{2}{|c|}{$\begin{array}{c}95 \% \text { de } \\
\text { intervalo de } \\
\text { confianza de la } \\
\text { diferencia }\end{array}$} \\
\hline & & & & & & & & & $\begin{array}{l}\text { Inferi } \\
\text { or }\end{array}$ & $\begin{array}{c}\text { Superi } \\
\text { or }\end{array}$ \\
\hline \multirow[t]{2}{*}{$\begin{array}{l}\text { Puntua- } \\
\text { ciones }\end{array}$} & $\begin{array}{l}\text { varianzas } \\
\text { iguales }\end{array}$ & $\begin{array}{r}0.88 \\
9\end{array}$ & .35 & 4.983 & 48 & 0.00 & 2.360 & 0.474 & 1.408 & 3.312 \\
\hline & $\begin{array}{l}\text { varianzas } \\
\text { desiguales }\end{array}$ & & & 4.983 & 48 & 0.00 & 2.360 & 0.474 & 1.408 & 3.312 \\
\hline
\end{tabular}

Fuente: Elaboración propia

En la tabla 6 se observó el valor de t_calculado es de 4,983 y es mayor que el valor de t_crítico 2,009, entonces, se tomó la decisión de rechazar la hipótesis nula y aceptar la hipótesis estadística planteada. También se observa que el valor del sig. bilateral o p- valor es 0,00 y es menor que el valor de significancia 0,025 , por la cual se decidió rechazar la hipótesis nula y aceptar la hipótesis estadística planteada. Concluyendo que existe evidencia estadística para inferir que existe efecto significativo al usar el Software Winstats en el aprendizaje de estadística, en los estudiantes de la especialidad de informática de la Facultad de Ciencias de la Universidad Enrique Guzmán y Valle, Perú.

\section{Hipótesis específica}

El planteamiento de la Hipótesis fue: Existe diferencia significativa entre el nivel de aprendizaje de estadística, en los estudiantes de la especialidad de informática de la Facultad de Ciencias en los grupos control y experimental después de haber aplicado el software Winstats. La hipótesis nula no existe diferencia significativa entre el nivel de aprendizaje de estadística, en los estudiantes de la especialidad de informática de la Facultad de Ciencias en los grupos control y experimental después de haber aplicado el software Winstats. El nivel de significancia 5\%, el nivel de confianza $95 \%$. Se eligió el estadístico tomando en cuenta que las varianzas son desconocidas, y desiguales; además $\mathrm{n}<=30$, entonces se aplicó la siguiente fórmula:

$$
\boldsymbol{t}_{c}=\frac{\bar{x}-\bar{y}}{\sqrt{\frac{(n-1) \boldsymbol{S}_{1}^{2}+(m-1) \boldsymbol{S}_{2}^{2}}{n+m-2} \sqrt{\frac{1}{n}+\frac{1}{m}}}}
$$

Donde: 
$\mathrm{T}_{\mathrm{c}}:$ : $\mathrm{t}$ " calculado

$\bar{X}_{1}$ : Promedio del primer grupo

$\bar{Y}_{2}$ : Promedio del segundo grupo

$S_{1}^{2}:$ Varianza del primer grupo

$S_{2}^{2}$ : Varianza del segundo grupo.

$\mathrm{n}$ : Tamaño de la muestra del primer grupo

$\mathrm{m}$ : Tamaño de la muestra del segundo grupo.

\section{Tabla 7}

Estadisticos por grupo. Diferencia significativa entre el nivel de aprendizaje de estadística, ambos momentos Postest_GC y Postest_GE

\begin{tabular}{llcccc}
\hline & \multicolumn{1}{c}{ Grupos } & $\boldsymbol{N}$ & Media & $\begin{array}{c}\text { Desviación } \\
\text { estándar }\end{array}$ & Media de error estándar \\
\hline Calificaciones & Experimental & 25 & 15,60 & 1.981 & 0.272 \\
& Control & 25 & 13,24 & 1.561 & 0.283 \\
\hline
\end{tabular}

Fuente: Elaboración propia

En la tabla 7 se observó las diferencias entre el nivel de aprendizaje de estadística, Grupo control Postest 13,24 y Grupo experimental Postest 15,60, después de aplicar Software Winstats, demostrándose que hay una diferencia significativa favorable al Postest Grupo Experimental. Esto significó que el nivel de aprendizaje en estadística mejoró en un nivel bueno después del experimento.

\section{Tabla 8}

Prueba de muestras independientes: diferencia significativa entre el nivel de aprendizaje de estadística, ambos momentos Postest_GC y Postest_GC

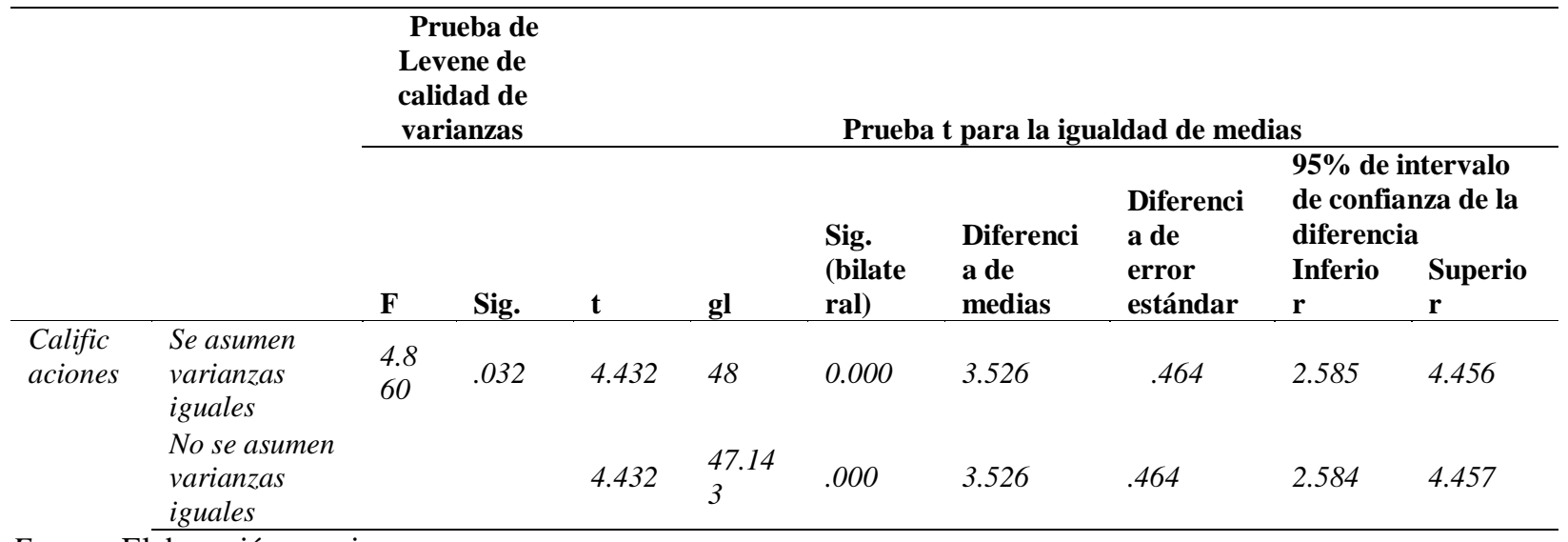

Fuente: Elaboración propia 


\section{Figura 2}

\section{Campana de dos colas los valores}

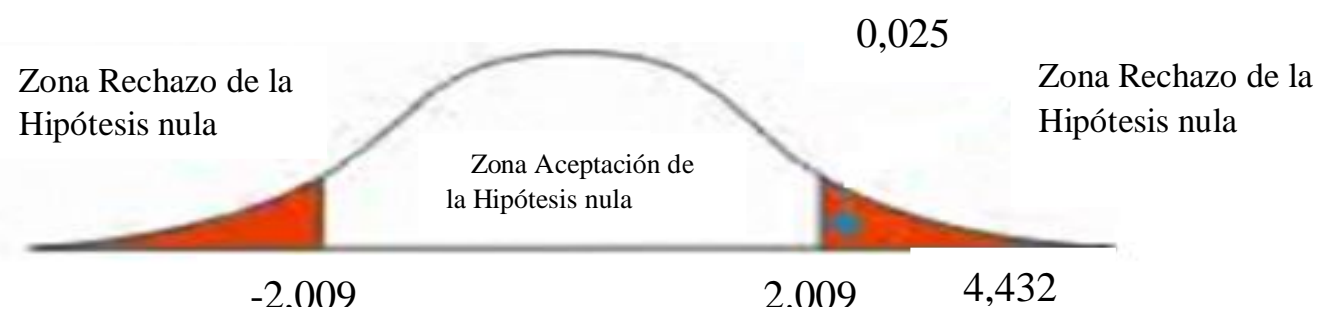

Nota: La figura muestra en la Campana de dos colas los valores del t- critico 2,009 y t- calculado 4,432, este último se localizó en la zona de rechazo de la hipótesis nula.

\section{Decisión}

Según la tabla 8 se observó que el valor de T- calculado es de 4,432, mayor que el valor de T-crítico 2,009, este último valor se obtuvo con los grados de libertad 48 y nivel de significancia 0,025 en la tabla de distribución de t- Student, entonces, se decidió rechazar la hipótesis nula y aceptar la hipótesis específica. También de la tabla 8 se observó el valor del sig bilateral 0,00 es menor que el valor de significancia 0,025, entonces se decidió rechazar la hipótesis nula y aceptar la hipótesis específica. En conclusión, se observó evidencia estadística para inferir que hubo diferencias significativas entre el nivel de aprendizaje de estadística, en los estudiantes de la especialidad de informática de la Facultad de Ciencias en los grupos control y experimental después de haber aplicado el software Winstats.

\section{Discusión}

La validez de contenido de los instrumentos de investigación fue evaluada por la técnica de juicio de expertos quienes calificaron y determinaron que si era aplicable a la investigación; el promedio que dieron fue de buena y muy buena, 89 y 90 respectivamente, es decir, que los ítems que se querían medir correspondían a los objetivos de la investigación, por lo cual se consideró que el instrumento era válido. Por otro lado, para ver si los resultados fueron confiables, se seleccionó una muestra piloto de 15 estudiantes que representaron el $60 \%$ de la muestra total, luego se administró las pruebas para cada momento Pre prueba y Pos prueba, con los datos recogidos se realizó la confiabilidad con la técnica de dos mitades sub técnica Spearman Brown, obteniéndose coeficientes de 0.85 y 0.86 respectivamente para cada instrumento, que está en escala muy alta de confiabilidad. Estos resultados permitieron tener la certeza que la medición con el instrumento sería confiable al tomar la muestra de estudio.

Para conocer la distribución de los datos, fue necesario evaluar con el método numérico de Shapiro Wilk por ser una muestra menor e igual a 50. El procesamiento de los datos se realizó con 
ayuda del software estadístico SPSS y se obtuvo el valor del Sig. bilateral 0.00, al comparar con el nivel de significancia 0,05, se decidió rechazar la hipótesis nula planteada que decía que los datos no tienen distribución normal, estos mismos resultados se dio en ambos instrumentos para la Pre prueba y Pos prueba. Se concluye que los datos si provienen de una distribución normal.

Para el contraste de hipótesis se utilizó la prueba paramétrica de t Student para muestras independientes; esta prueba fue la más indicada debido a que los datos tuvieron una distribución normal, la muestra fue menor que 30 y variables cuantitativas. Con respecto a la prueba general procesados con ayuda del software SPSS el resultado del sig. Bilateral o p-valor fue menor que el nivel de significancia 0.025 , de acuerdo con estos resultados se afirma que existió evidencia estadística para rechazar la hipótesis nula, demostrando el efecto de la variable Winstats sobre la variable aprendizaje de estadística.

\section{Conclusiones}

La aplicación del Software Winstats mejoró significantemente el proceso de enseñanzaaprendizaje de estadística. En los estudiantes mejoró sus competencias, capacidades, habilidades y actitudes. En los docentes su metodología, su estrategia y un cambio paradigmático al hacer uso de las tecnologías de la información y comunicación.

A partir de los resultados obtenidos al 95\% de nivel de confianza se concluye que, sí hubo efecto significativo del Software Winstats en el aprendizaje de estadística, en los estudiantes de la especialidad de informática de la Facultad de Ciencias de la Universidad Enrique Guzmán y Valle, esto se evidenció con el valor de significancia sig. bilateral 0.01 es menor que 0,05 nivel de significancia. En cuanto a las hipótesis específicas 1 y 2 al 95\% de nivel de confianza el software Winstats tuvo efecto en el aprendizaje de estadística en su dimensión conceptual, procedimental. Finalmente, teniendo como base la tercera hipótesis específica de la investigación se concluyó al 95\% de nivel de confianza que si existió diferencias significativas entre el nivel de aprendizaje de estadística en los grupos control y experimental después de aplicar el Winstats.

\section{Referencias bibliográficas}

Antonio, V. (2002). Dificultades de aprendizaje. Valencia. España. Editorial Promolibro. Ausubel (1970). Aprendizaje Significativo. México. Edit. Trillas

Anderson, D., Sweeney, D., y Williams, T. (2008). Estadística para administración y economía. loa Edición. México. https://www.upg.mx/wp-content/uploads/2015/10/libro-13Estadistica-para-administracion-y-economia.pdf

Álvarez, R. (2012). Facilitación y Capacitación virtual en América Latina. Colombia: Editorial Travesías.

Álvarez, L. y Solís, K. (2018). Uso de GeoGebra y el aprendizaje de la estadística descriptiva para estudiantes del cuarto grado de educación secundaria de la Institución Educativa Daniel Alcides Carrión de Cerro de Pasco. http://repositorio.undac.edu.pe/bitstream/undac/1543/1/T026_46258318_T.pdf 
Winstats en el aprendizaje de estadística en los estudiantes de informática de la Universidad Enrique Guzmán y

Valle-Perú

Bauman, Z. (2013). La sociedad individualizada. México. Edit. Ediciones Catedra

Bruner, J. (2003). La Importancia de la Educación. España. Editorial Paidós Ibérica.

Banet, M. (2001). Tecnología en la Enseñanza Abierta. México. Edit. Trillas

Campo, E. (2020). Estadística descriptiva multivariada. 1ra. Edición. Bogotá. Por la Universidad Nacional de Colombia.

Caballero, L., Quivio, R., Morales, G., Cajavilca, P., Gutiérrez, S. y Vargas, J. (2016). Estadística aplicada a la investigación con SPSS. Perú. Edición Kindle

Chistensen, H. (2013). Estadística paso a paso. México: 3era edición. Edit. Trillas.

Calero, M. (1997). Constructivismo: un reto de innovación pedagógica. Perú

Editorial. San Marcos.

Carrasco, S. (2009). Metodología de la investigación científica. Perú. Lima. Segunda reimpresión. Edit. San Marcos.

Castells, M. (2000). The Information Age: Economy, Society and Culture. Vol. 1: The Rise of the Network Society; Vol. 2: The Power of Identity; Vol. 3: End of Millennium, Blackwell, Oxford

Campistrous, L. y Rizo, C. (1996). Aprende a Resolver Problemas Aritméticos. La Habana: Pueblo y Educación.

Cabero, J. (2001): Las tecnologías de la información y comunicación en la Universidad. Sevilla.

De La Horra, J. (2013). Estadística aplicada. Brasil. Ediciones Díaz de santos.

Estrada, A. (2002). Análisis de las actitudes y conocimientos estadísticos elementales en la formación del profesorado. Tesis doctoral. Universidad Autónoma de Barcelona. http://www.tdx.cat/tdx-0502103-191818

Flores. P. (2017) Uso del Software Wiris y su Efecto en el Aprendizaje de límites e integrales en el curso de Matemática II en los estudiantes de Ingeniería Industrial del segundo ciclo de la Universidad César Vallejo. https://repositorio.une.edu.pe/handle/une/1554

Fuenlabrada, S. (2008). Probabilidad y Estadística. Tercera edición. México. Editorial Mc GrawHill.

Gamero, C. (2017). Estadística I. Elementos de estadística descriptiva y de la teoría de probabilidad. 1ra. Edición. España. colecciones manuales

García, R. (2013). Inferencia Estadística y Diseño de Experimentos. Argentina. Editorial Universitaria de Buenos Aires.

Garrido, M. (2013), en su estudio titulado: El método de resolución de problemas de George Polya en el desarrollo de capacidades de Estadística en los alumnos de 4to. grado de Educación Básica Regular 6023 Julio C. Tello Lurín

Garzo, F. y García, F. (2014) Estadística. España: Mc Graw Hill Interamericana.

Gómez, A. (2017). Proceso de Enseñanza y Gestión Participativa (2a ed.). República Dominicana: Universidad Tecnológica del Cibao Oriental. Uteco.

Guerrero, M. (2014). Metodologías activas y aprendizaje por descubrimiento. Las TIC y la Educación. Marpadal Interactive Media.

Hidalgo, L. (2005). Validez y confiabilidad en la investigación cualitativa. México: Mc Graw Hill Hernández, R., Fernández, C. y Baptista, P. (2014). Metodología de la investigación ( $6^{\circ}$ ed.). México: Ediciones McGraw Hill. 
Hurtado, J. (2012). Metodología de la investigación: guía para una comprensión holística de la ciencia (4a. ed.). Bogotá-Caracas: Ciea-Sypal y Quirón

Mendenhall, W., Beaver, R., y Beaver, B. (2010). Introducción a la probabilidad y estadística $13 a$ Edición. México. http//www.latinoamericana.cengage.com.

Medina, Z. (2019), Aplicación del software SPSS en el proceso de enseñanza - aprendizaje de estadística en los estudiantes de la Facultad de Ciencias de la Comunicación, Turismo y Psicología. https://repositorio.usmp.edu.pe/handle/20.500.12727/5883

Murillo, J. y Castellanos, R. (2011). Interactividad y atención a la diversidad en el aprendizaje de la estadística. Enseñanza de las ciencias: revista de investigación y experiencias $\begin{array}{llllll}\text { didácticas. } & \text { Vol. } & 29, & \text { n. } & \text { (3), } & \text { pp. 381-02. }\end{array}$ https://raco.cat/index.php/Ensenanza/article/view/247887.

Nagel, E. y Newman, P. (1979). El Teorema de Gödel. Madrid. Editorial Tecnos.

Pardo, A. y Ruiz, M. A. (2002). SPSS. Guía para el análisis de datos. Madrid: McGraw-Hill.

Prieto, Gerardo; Delgado, Ana R. (2010). Fiabilidad y validez. Papeles del psicólogo. España: Consejo General de Colegios Oficiales de Psicólogos.

Popper, K. (1974). El Conocimiento Objetivo. Madrid. Editorial Tecnos.

Parris, R. (1996). Software Winstats. España. Mc Graw Hill Interamericana

Rustom, A. (2012). Estadística descriptiva, probabilidades. Una visión conceptual y aplicada. Universidad de Chile. http://www.agren.cl/estadistica.

Salazar, C. y Santiago del Castillo, G. (2018): Elementos básicos de la estadística. 1ra. Edición. Ecuador.

Sánchez, J. (2003). Aprendizaje visible, tecnología invisible. Santiago de Chile: Domen ediciones.

Sote, A. (2013) Principios de Estadística. Caracas: Venezuela Editorial Panapo

Santos, M. (2008). On the use of technology to represent and explore mathematical objects or problems dynamically. Mathematics and Computer Education.

Sinclair, N. y Yurita, V. (2008). To be or to become: How dynamic geometry changes discourse. Research in Matematices Education,

Sánchez, C. (2007) Las funciones un paseo por su historia. Nivola. Colección Ciencia abierta.

Torres, L. (2019) SIMSTAT en el aprendizaje de estadística en los estudiantes de la Universidad Nacional de Educación en Lima Perú. http://repositorio.une.edu.pe/handle/UNE/3564

Vílchez C, Vara A. (2009). Manual de redacción de artículos científicos. Lima: Universidad de San Martin de Porras.

Winstats, (2018). Software statistic libre. Soft Warehouse. User Manual. 